\title{
An unusual cause of chest pain
}

\author{
Simon A Brown, Sanjay Popat, Robert Carr
}

A 66-year-old woman was admitted with ischaemic chest pain. She had a 15-year history of hypertension, was an ex-smoker and was taking prednisolone $7 \mathrm{mg}$ daily for polymyalgia rheumatica (diagnosed two years earlier). A subendocardial myocardial infarction was diagnosed on the basis of electrocardiographic (ECG) changes and raised creatine kinase (MB fraction 14 $\mathrm{U} / 1[0-10])$. A full blood count showed haemoglobin $12.2 \mathrm{~g} / \mathrm{dl}$, white cell count $9.8 \times 10^{9} / 1$, and platelet count $107 \times 10^{9} / 1$. Renal function was normal (creatinine $72 \mu \mathrm{mol} / \mathrm{l}$ ). Aspirin, $150 \mathrm{mg}$ daily, was commenced.

During the first five days the patient had a mild pyrexia (up to $37.7^{\circ} \mathrm{C}$ ). On the fifth day she suffered recurrent ischaemic chest pain followed by a brief episode of unconsciousness. The patient recovered with no residual neurological signs. Platelet count was $10 \times 10^{9} / 1$. A similar episode of unconsciousness occurred two days later. On examination she had widespread bruising. As well as a persistent thrombocytopenia (platelets $24 \times 10^{9} / 1$ ) she was found to be anaemic (haemoglobin $7.1 \mathrm{~g} / \mathrm{dl}$ ) and the blood film also showed red cell fragmentation (figure). The direct antiglobulin test was negative and the coagulation screen was normal (International Normalised Ratio 1.1, activated partial thromboplastin time ratio 0.78 , and fibrinogen $5.73 \mathrm{~g} / 1$ [1.5-4.0]). A bone marrow aspirate showed increased numbers of megakaryocytes.

Department of

Haematology,

St Thomas' Hospital, London, UK

SA Brown

S Popat

R Carr

Correspondence to SA Brown, Department of Haematology,

University Hospital of

Wales, Heath Park

Cardiff CF4 4XW, UK

Accepted 1 May 1997

\section{Questions}

1 What is the diagnosis?

2 How should this patient be managed?

Figure Peripheral blood film 


\section{Answers}

QUESTION 1

The diagnosis is thrombotic thrombocytopenic purpura (TTP). TTP is characterised by the pentad of thrombocytopenia, micro-angiopathic haemolytic anaemia, fever, renal dysfunction and fluctuating neurological deficits. This classical pentad is present in only $40 \%$ of patients at diagnosis and patients often have nonspecific symptoms, eg, malaise, nausea and abdominal pain. ${ }^{1}$ The differential diagnosis consists of conditions in which thrombocytopenia and micro-angiopathic haemolytic anaemia occur (box 1). The diagnosis is usually made from the clinical setting and the result of the coagulation screen, which is normal in the majority of cases. Fibrin degradation products can be elevated, as seen in disseminated intravascular coagulation.

\section{QUESTION 2}

TTP was in the past associated with an invariably fatal outcome. Therapy with plasma exchange has transformed the prognosis with up to $90 \%$ of patients responding to therapy. ${ }^{2}$ Initial therapy can be started with infusions of fresh frozen plasma at $30 \mathrm{ml} / \mathrm{kg} /$ day. Glucocorticoids are usually started immediately, either methylprednisolone or prednisolone. As soon as plasma exchange can be started this should be commenced at 3 to 41 /day with fresh frozen plasma. Anaemia is treated with red cell transfusions but platelet transfusions are best avoided unless there is life-threatening haemorrhage, due to the risk of thrombosis. Additional therapeutic modalities include vincristine, antiplatelet drugs (aspirin and/or dipyridamole), plasma exchange with cyrosupernatant, splenectomy, prostacyclin and, recently, a case of refractory TTP was successfully treated with a solvent/detergenttreated plasma. ${ }^{3}$ Therapy can be monitored by measurement of haemoglobin, platelet count, reticulocyte count, and serum lactate dehydrogenase concentration, and if ultra-large von Willebrand multimers are present at diagnosis these can be assessed serially. ${ }^{3}$ Treatment is continued and then gradually reduced after normalisation of the clinical and laboratory defects. Over $50 \%$ of patients have normal platelet counts and lactate dehydrogenase levels after three to nine days of plasma exchange. Following successful treatment of

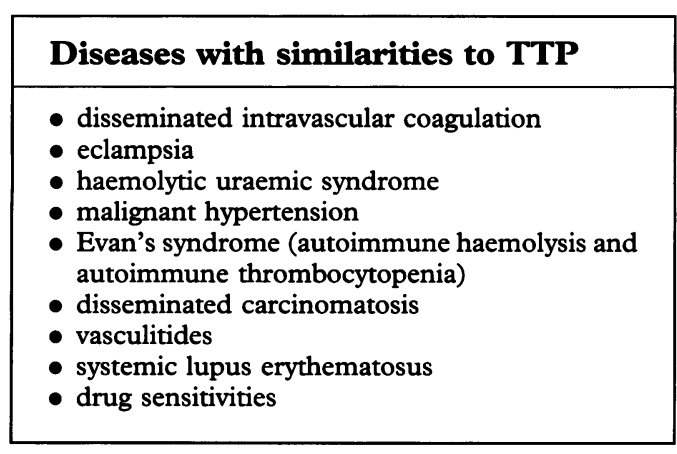

Box 1
Learning / summary points

- thrombotic thrombocytopenic purpura is characterised by thrombocytopenia and micoangiopathic haemolytic anaemia. Other features are fever, neurological disturbances and renal impairment

- therapy with plasma exchange has transformed the prognosis of this disease, with $90 \%$ of patients responding to treatment

- successful treatment is associated with relapse in a third of cases

- investigation of a thrombocytopenia requires examination of the peripheral blood film

Box 2

acute TTP it has been recognised that up to $30 \%$ of patients relapse. ${ }^{4}$

\section{Discussion}

TTP is a rare disorder, with an incidence of 1 per 50000 hospital admissions. ${ }^{1}$ It is a multisystem disorder characterised by widespread microvascular microthrombi composed predominantly of platelets. The platelet aggregates seem to be associated with the excessive release of ultra-large von Willebrand multimers, probably as a result of a systemic endothelial cell disturbance. In most cases of TTP no precipitating factor can be identified, but there are a few well documented risk factors which include, pregnancy, AIDS, mitomycin C and autoimmune disorders.

This patient presented with chest pain, ECG changes and later an echocardiogram revealed antero-apical and inferior hypokinesia of the left ventricle. The patient made a complete recovery after eight weeks treatment, during which time she received 441 units (approximately $99.5 \mathrm{l}$ ) of fresh frozen plasma. The low incidence of chest pain at presentation (3\%) does not correlate with pathological studies which show myocardial haemorrhage and necrosis in over $50 \%$ of cases coming to autopsy. ${ }^{5}$ Cardiac decompensation is usually precipitated by worsening anaemia, although cardiac conduction defects have been documented. ${ }^{5}$ Despite this frequent involvement there are few cases of clinically documented myocardial damage, ${ }^{6}$ and none with a successful outcome. Review of the original full blood count showed micro-angiopathic haemolytic anaemia with a mild thrombocytopenia, suggesting that the onset of TTP preceded the myocardial damage in this case. Recognition of thrombocytopenia with micro-angiopathic haemolytic anaemia allowed a diagnosis of TTP to be made and appropriate therapy to be instituted.

\section{Final diagnosis}

Thrombotic thrombocytopenic purpura presenting with chest pain.

Keywords: thrombotic thrombocytopenic purpura, micro-angiopathic haemolytic anaemia, ultra-large von Willebrand multimers 
1 Ridolfi RL, Bell WR. Thrombotic thrombocytopenic purpura. Report of 25 cases and review of the literature. Medicine 1981; 60: 413-28.

2 Bell Wr, Braine HG, Ness PM, Kuckler TS. Improved survival in thrombotic thrombocytopenic purpura-hemolytic uremic syndrome: clinical experience in 108 patients $N$ Engl f Med 1991; 325: 398-403.

3 Harrison CN, Lawrie AS, Iqbal A, Hunter A, Machin SJ. Hasma exchange with solvent/detergent - treated plasma of resistant thrombotic thrombocytopenic purpura. $B r \quad f$ Haematol 1996; 94: 756-8.
4 Rose M, Eldor A. High incidence of relapses in thrombotic thrombocytopenic purpura. Am $\mathcal{F}$ Med 1987; 83: 437-44. 5 Ridolfi RL, Hutchins GM, Bell WR. The heart and cardiac conduction system in thrombotic thrombocytopenic purpura. A clinicopathological study of 17 autopsied patients. Ann Intern Med 1979; 9: 357-63.

6 Eagle KA, Fallon JT. A 41-year-old woman with thrombocytopenia, anemia, and sudden death. $N$ Engl $f$
Med 1994; 331: $661-7$.

\title{
Epigastric pain and a left upper quadrant mass in an elderly woman
}

\author{
R Joarder, AC Harris, M Gibson, A Al-Kutoubi
}

An 81-year-old woman presented to her general practitioner complaining of epigastric discomfort radiating to her back. This was associated with weakness, fatigue, diarrhoea, and a microcytic anaemia (haemoglobin $8.6 \mathrm{~g} / \mathrm{dl}$ ). On examination, she was found to have a smooth mass in the left hypochondrium. Upper gastrointestinal tract endoscopy was normal. An abdominal ultrasound scan showed an $11 \times 7 \times 5 \mathrm{~cm}$ mass in the left hypochondrium which had air running through it. The liver, spleen and kidneys appeared normal. A computed tomography (CT) scan was performed (figure 1).

\section{Department of Diagnostic Radiol- ogy, St Mary's NHS Trust Hospital, Praed Street, London W2 1NY, UK R Joarder AC Harris M Gibson \\ A Al-Kutoubi}

Accepted 16 April 1997

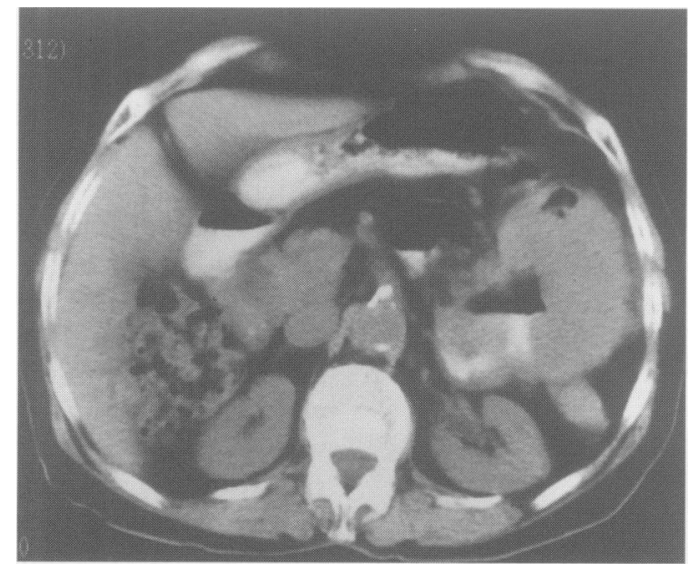

\section{Questions}

1 What are the findings on the CT scan (figure 1)?

2 What would be your next investigation? 3 What is the differential diagnosis?
Figure 1 CT scan through the upper abdomen following oral and intravenous contrast 